\title{
An X-ray and optical study of the cluster A33
}

\author{
S. Colafrancesco ${ }^{1}$, C.R. Mullis ${ }^{2}$, A. Wolter ${ }^{3}$, I.M. Gioia ${ }^{2,10,11}$, T. Maccacaro ${ }^{3}$, A. Antonelli ${ }^{1}$, F. Fiore ${ }^{1}$, J. Kaastra ${ }^{4}$, \\ R. Mewe ${ }^{4}$, Y. Rephaeli ${ }^{5}$, R. Fusco-Femiano ${ }^{6}$, V. Antonuccio-Delogu ${ }^{7}$, F. Matteucci ${ }^{8}$, and P. Mazzotta ${ }^{9}$ \\ 1 Osservatorio Astronomico di Roma via dell'Osservatorio 2, I-00040 Monteporzio, Italy \\ e-mail: cola@coma.mporzio.astro.it \\ 2 Institute for Astronomy, University of Hawaii, 2680 Woodlawn Drive, Honolulu, HI 96822, U.S.A. \\ 3 Osservatorio Astronomico di Brera, Via Brera 26, Milano, Italy \\ 4 SRON, Sorbonnelaan 2, 3584 CA Utrecht, The Netherlands \\ 5 School of Physics and Astronomy, Tel Aviv University, 69978, Israel \\ 6 IAS - CNR, Via Fosso del Cavaliere, I-00133 Roma, Italy \\ 7 Osservatorio Astrofisico di Catania, Via A. Doria, Catania, Italy \\ 8 Osservatorio Astronomico di Trieste, Via dell'Osservatorio, Trieste, Italy \\ 9 Dipartimento di Fisica, Università di Roma "Tor Vergata", Via della Ricerca Scientifica 1, I-00133 Roma, Italy \\ 10 Home institution: Istituto di Radioastronomia del CNR, Via Gobetti 101, I-40129 Bologna, Italy \\ 11 Visiting Astronomer at the W.M. Keck Observatory, jointly operated by the California Institute of Technology, the University \\ of California and the National Aereonautics and Space Administration
}

Received February 18, 1999; accepted January 4, 2000

\begin{abstract}
We report the first detailed X-ray and optical observations of the medium-distant cluster A33 obtained with the Beppo-SAX satellite and with the UH $2.2 \mathrm{~m}$ and Keck II telescopes at Mauna Kea. The information deduced from X-ray and optical imaging and spectroscopic data allowed us to identify the X-ray source 1SAXJ0027.2-1930 as the X-ray counterpart of the A33 cluster. The faint, $F_{2-10 \mathrm{keV}} \approx 2.410^{-13} \mathrm{erg} \mathrm{s}^{-1} \mathrm{~cm}^{-2}$, X-ray source 1SAXJ0027.2-1930, 2 arcmin away from the optical position of the cluster as given in the Abell catalogue, is identified with the central region of A33. Based on six cluster galaxy redshifts, we determine the redshift of A33, $z=0.2409$; this is lower than the value derived by Leir \& Van Den Bergh (1977). The source X-ray luminosity, $L_{2-10 \mathrm{keV}}=7.710^{43} \mathrm{erg} \mathrm{s}^{-1} \mathrm{~cm}^{-2}$, and intracluster gas temperature, $T=2.9 \mathrm{keV}$, make this cluster interesting for cosmological studies of the cluster $L_{\mathrm{X}}-T$ relation at intermediate redshifts. Two other X-ray sources in the A33 field are identified. An AGN at $z=0.2274$, and an M-type star, whose emissions are blended to form an extended X-ray emission $\sim 4$ arcmin north of the A33 cluster. A third possibly point-like X-ray source detected $\sim 3$ arcmin north-west of A33 lies close to a spiral galaxy at $z=0.2863$ and to an elliptical galaxy at the same redshift as the cluster.
\end{abstract}

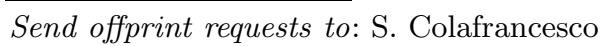

Key words: galaxies clusters: individual: A33 - Cosmology: observations - galaxies: active observations: X-rays — intergalactic medium

\section{Introduction}

A33 is a medium-distant Abell cluster of galaxies with very few and sparse information in both the X-ray and the optical bands. This cluster was claimed to have been detected by the HEAO1-A1 all sky survey (Johnson et al. 1983; Kowalski et al. 1984) with a count rate of $3.77 \pm 0.47$ counts $\mathrm{cm}^{-2} \mathrm{~s}^{-1}$ in the $2-6 \mathrm{keV}$ energy band. Its luminosity was estimated, with large uncertainties, to be $L_{2-6 \mathrm{keV}} \approx 2.3410^{45} \mathrm{erg} \mathrm{s}^{-1}$.

A33 was also observed with the GINGA LAC detector from December 9 to December 10, 1988 (Arnaud et al. 1991), but no X-ray emission was found at the optical position of the cluster. From such a non-imaging observation, Arnaud et al. (1991) were able to put an upper limit on the luminosity of A33, $L_{2-10} \mathrm{keV}<610^{44} \mathrm{erg} \mathrm{s}^{-1}$, assuming a temperature $T=8.4 \mathrm{keV}$. The value of the $\mathrm{X}$-ray luminosity derived from GINGA data is inconsistent with the one derived from the HEAO1-A1 observation (note, however, that A33 lies at the edge of the error box for the position of the HEAO1 source).

The source 1RXSJ002709.5 - 192616 in the ROSAT Bright Source Catalog (BSC: Voges et al. 1996), at coordinates $\alpha_{2000}^{x}=00^{\mathrm{h}} 27^{\mathrm{m}} 09.50^{\mathrm{s}}$ and $\delta_{2000}^{x}=-19^{\circ} 26^{\prime} 16^{\prime \prime}$, 
has been observed for $317 \mathrm{~s}$ with a count rate of $0.062 \pm$ $0.017 \mathrm{cts} / \mathrm{s}$. This source has 19.6 net counts in the $0.1-$ $2.4 \mathrm{keV}$ energy band corresponding to a flux $F_{0.2-2.4}=$ $(9.3 \pm 2.6) 10^{-13} \mathrm{erg} \mathrm{s}^{-1} \mathrm{~cm}^{-2}$ (assuming a nominal conversion factor of $\left.1.510^{-11} \mathrm{erg} \mathrm{cm}^{-2} \mathrm{~s}^{-1} \mathrm{cts}^{-1}\right)$ and does not appear to be extended. This source is unrelated to the cluster and most probably associated with an AGN which is only $5.4^{\prime \prime}$ away (see Table 1, source 1SAXJ0027.1-1926, and Table 2, source A).

In the optical band there is no detailed information except from that derived from the extensive study of Leir \& Van Den Bergh (1977), who classified A33 as a distance class $D=6$, richness $R=1$, Bautz-Morgan-class-III cluster. In the Abell (1958) catalog, A33 has 69 galaxies which lie within one Abell radius (2.7 $z^{-1}$ arcmin) and which are not more than 2 mag fainter than the third brightest galaxy. Its photometrically estimated redshift, $z=0.28$, was derived by Leir \& Van Den Bergh (1977) from the cluster optical diameter and the magnitude of the brightest and tenth-brightest cluster galaxies.

In this paper we present a new X-ray observation of A33 obtained with Beppo-SAX. This observation enables us to derive detailed information on the X-ray source, on its morphology and thermal properties. The complex appearance of the X-ray emission in the field of A33 prompted us to obtain optical images and spectroscopic information for several objects in the field.

The plan of the paper is the following. In Sect. 2 we present the basic information on the Beppo-SAX observation and data reduction. In Sect. 3 we describe the optical data and in Sect. 4 we discuss the X-ray spectroscopy of the various sources in the A33 field. We summarize our results for A33 and discuss their implications in Sect. 5.

Throughout the paper $H_{0}=50 \mathrm{~km} \mathrm{~s}^{-1} \mathrm{Mpc}^{-1}$ and $\Omega_{0}=1$ are used unless otherwise noted.

\section{Beppo-SAX observation}

The A33 field was observed with the Narrow Field Instruments (NFI) of the Beppo-SAX satellite from November $23^{\text {th }}$ to $25^{\text {th }}, 1996$. The total effective exposure time is $t_{\exp }=3.841710^{4} \mathrm{~s}$ for the LECS instrument and $t_{\exp }=7.761010^{4} \mathrm{~s}$ for the MECS instrument (see e.g. Boella et al. 1997a and 1997b for a technical description of the Beppo-SAX mission and instrumentation).

Data preparation and linearization was performed using the SAXDAS v.1.3 package under the FTOOLS environment. The imaging analysis was performed using the XIMAGE package (Giommi et al. 1991). The extraction of the source and background spectra was done within the XSELECT package. The spectral analysis was performed using XSPEC v.9.0.

The only previous claimed X-ray detection of A33 was done with the HEAO1 satellite (Johnson et al. 1983; Kowalski et al. 1984). Due to the large error box of the
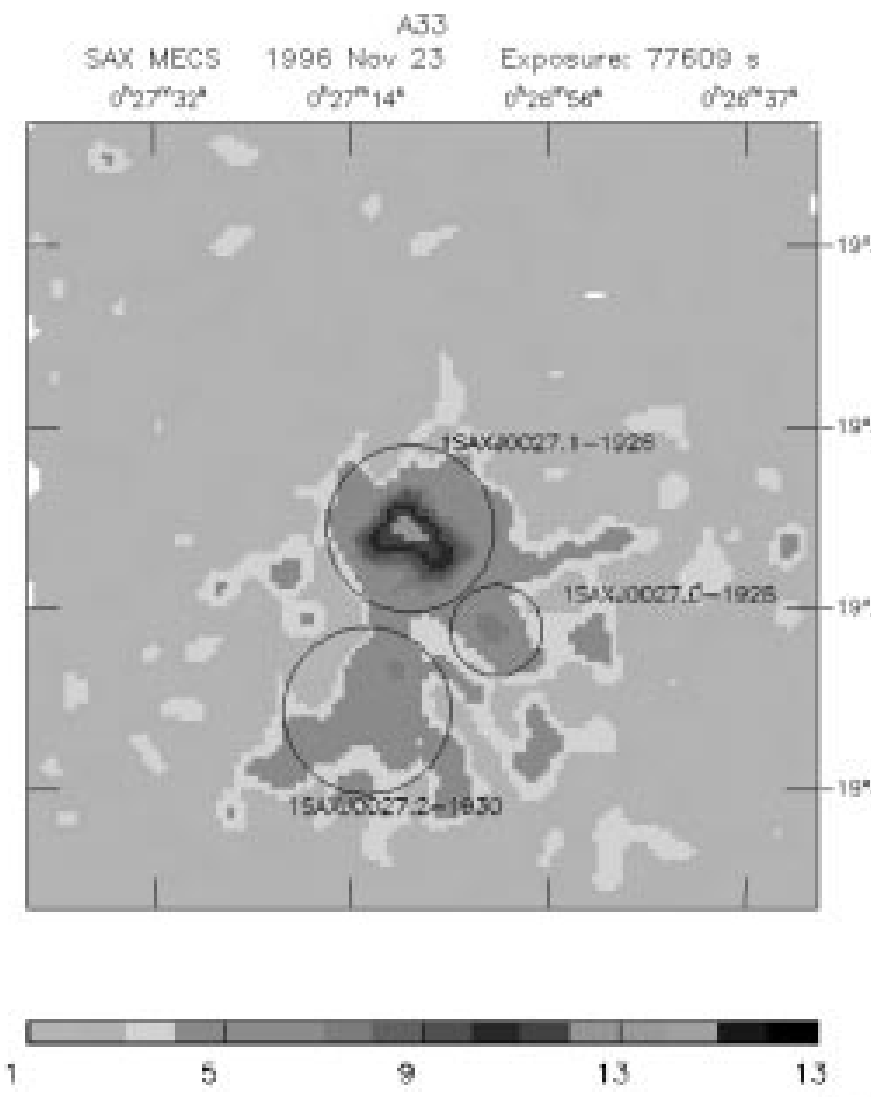

Fig. 1. The Beppo-SAX image of A33 in the $2-10 \mathrm{keV}$ energy band. The three different components of the emission are labeled according to the text. The circles indicate the extraction area for each X-ray source. Note that 1sSAXJ0027.2-1930 has also a diffuse, low-surface brightness distribution which appears to be extended in the southern part of the image. The image has been deconvolved with a wavelet transform using a smoothing length of 3.5 pixels ( 1 pixel $=8$ arcsecs). North is up and East to the left

HEAO1 detectors, the coordinates of the X-ray source were associated with the optical coordinates of the A33 cluster. Thus the Beppo-SAX observation was centered on the optical coordinates $\alpha_{2000}^{\circ}=00^{\mathrm{h}} 26^{\mathrm{m}} 52.7^{\mathrm{s}}$ and $\delta_{2000}^{\circ}=-19^{\circ} 32^{\prime} 29^{\prime \prime}$. The MECS $2-10 \mathrm{keV}$ X-ray image of the field is shown in Fig. 1, where three different subsystems are evident: a bright and apparently extended source, 1SAXJ0027.1-1926, an extended but smaller source, 1SAXJ0027.2-1930, located to the south of the brightest source and an apparently point-like source, 1SAXJ0027.0-1928, located to the west. Positions, count rates and extraction region radii, $R_{\text {extr }}$, are listed in Table 1. The sources have sufficient count rates to be detected individually at more than 4 sigma level by the MECS instruments. The poorer spatial resolution of the LECS instead allows only to determine the count rate of the brightest source 1SAXJ0027.1-1926. In the following 
Table 1. LECS $(0.1-2 \mathrm{keV})$ and MECS $(2-10 \mathrm{keV})$ count rates

\begin{tabular}{ccccccc}
\hline \hline Source & $\alpha_{2000}$ & $\delta_{2000}$ & $t_{\exp }$ & Count rate & Count rate & $R_{\text {extr }}$ \\
\hline & $\left({ }^{\mathrm{h} \mathrm{m} \mathrm{s}}\right)$ & $\left({ }^{o}{ }^{\prime \prime \prime}\right)$ & $(\mathrm{s})$ & $\begin{array}{c}\left(10^{-3} \mathrm{~s}^{-1}\right) \\
\text { LECS }\end{array}$ & $\begin{array}{c}\left(10^{-3} \mathrm{~s}^{-1}\right) \\
\text { MECS }\end{array}$ & arcmin \\
& & & & $7.6 \pm 0.7$ & $7.8 \pm 0.6$ & 2 \\
\hline 1SAXJ0027.1-1926 & 002708 & -192638 & 77609 & $7.6 \pm 0.76$ & 2 \\
1SAXJ0027.2-1930 & 002712 & -193032 & 77609 & - & $1.76 \pm 0.23$ & $1.14 \pm 0.19$ \\
1SAXJ0027.0-1928 & 002701 & -192830 & 77609 & - & 1 \\
\hline \hline
\end{tabular}

we describe the spatial structure of each source detected in the A33 field as derived from the MECS data.

The MECS PSF is $\approx 1$ arcmin Half Energy Width, and this spatial resolution allows us to detect the sources 1SAXJ0027.1-1926 and 1SAXJ0027.2-1930 as extended in the MECS image of Fig. 1.

The source 1SAXJ0027.1-1926 has an extension of $\sim 2$ arcmin (radius). As discussed in Sects. 3 and 4, this source is most probably the result of the blending of two point-like sources not resolved by the MECS PSF. The X-ray MECS image contours superposed onto the POSS II image of the field plotted in Fig. 2 show that there is no clear galaxy excess associated to the X-ray source 1SAXJ0027.1-1926.

The source 1SAXJ0027.2-1930, located $\sim 4.5$ arcmin south of the brightest source (see Fig. 1), has an extension of $\gtrsim 1.5$ arcmin radius. Using a $\beta$-model with values $\beta=0.75$ and $r_{\mathrm{c}}=260 \mathrm{kpc}\left(H_{0}=50, \Omega_{0}=1\right)$ chosen as representative of such low luminosity objects, and convolved with the MECS PSF we find a central density of $\approx 3.910^{-3} \mathrm{~cm}^{-3}$. Moreover, an extended, low surface brightness X-ray emission is visible in the southern part of the image (see Figs. 1 and 2). Such a low surface brightness source extends for a few arcminutes at levels of $\sim 10^{-4}$ cts s $^{-1} \mathrm{~cm}^{-2} \operatorname{arcmin}^{-2}$. The extended source 1SAXJ0027.2-1930 is associated with A33 as shown in the POSS II image of the field (see Fig. 2 and Sect. 3).

The third source 1SAXJ0027.0-1928, located $\sim 4$ arcmin south-west of the brightest source, has a point-like appearance. Two faint objects in the POSS II are positionally consistent with 1SAXJ0027.0-1928.

\section{Optical imaging and spectroscopy}

Due to the lack of detailed optical information in the literature for A33, we took $I$ and $B$ images of the cluster region on November 23 and 241997 at the Keck II telescope. The images were obtained using the Low-Resolution and Imaging Spectrograph (LRIS) (Oke et al. 1995) in imaging mode, resulting in a scale of $0.215^{\prime \prime}$ pixel $^{-1}$ and a field of view of $6^{\prime} \times 7^{\prime}$.3. The $I(B)$ images were taken in $0.4^{\prime \prime}-0.5^{\prime \prime}$ seeing on the first night and consist of $3 \times 300 \mathrm{~s}(4 \times 120 \mathrm{~s})$ dithered exposures centered at $\alpha=00^{\mathrm{h}} 27^{\mathrm{m}} 10.5$ and $\delta=-19^{\circ} 29^{\prime} 18^{\prime \prime} \quad(\mathrm{J} 2000)$, the southern region of the $\mathrm{X}$-ray emission complex. On the second

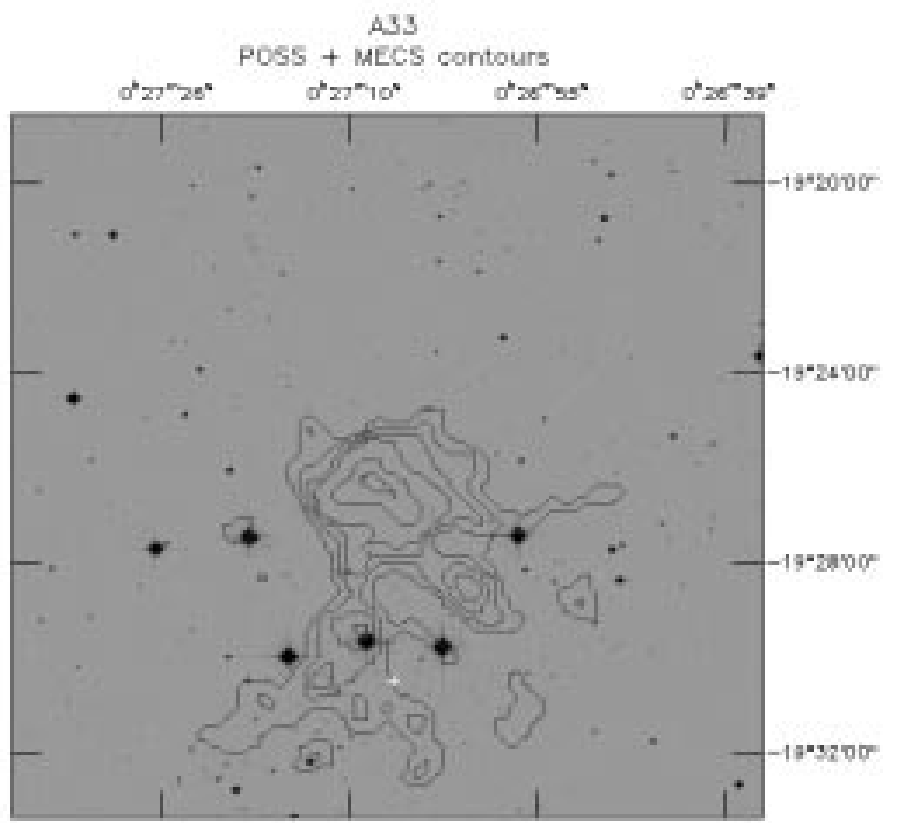

Fig. 2. The optical image of A33 taken from the POSS II plate and the X-ray contours of the Beppo-SAX image obtained with the MECS detector in the $2-10 \mathrm{keV}$ energy band. Contours are taken from the image shown in Fig. 1 and are logarithmically spaced. The image has been deconvolved with a wavelet transform using a smoothing length of 3.5 pixels $(1$ pixel $=$ 8 arcsecs). The white cross indicates the position of A33 from the Abell catalogue. The first X-ray contour is at $3 \sigma$ from the background level. North is up and East to the left

night $\left(0.8^{\prime \prime}\right.$ seeing $)$ we took $2 \times 120 \mathrm{~s} I(2 \times 300 \mathrm{~s} B)$ exposures centered at $\alpha=00^{\mathrm{h}} 27^{\mathrm{m}} 09.8$ and $\delta=-19^{\circ} 26^{\prime} 12^{\prime \prime} .4$ (J2000), the northern region of the X-ray emission system. The optical position of A33 (Fig. 2) is close to an open stellar cluster. Figure 3 shows the $B$ images for both North (Fig. 3a) and South (Fig. 3b) regions. No excess of galaxies is present in the northern region at the position of 1SAXJ0027.1-1926 (Fig. 3a), while Fig. 3b reveals an overdensity of galaxies in the region of the X-ray source 1SAXJ0027.2-1930.

Spectroscopic observations for several objects in the field were carried out on August 16, 17 and 19, 1998, with the Wide Field Grism Spectrograph and the Tek2048 × 2048 CCD attached to the University of Hawaii $2.2 \mathrm{~m}$ telescope on Mauna Kea. We used the $420 \mathrm{l} / \mathrm{mm}$ grating which provided $\mathrm{a} \sim 3990-9900 \AA$ coverage and a pixel 
Table 2. Optical results

\begin{tabular}{|c|c|c|c|c|}
\hline Name & $\begin{array}{c}\alpha_{2000} \\
\left({ }^{\mathrm{h} \mathrm{m} \mathrm{s}}\right)\end{array}$ & $\begin{array}{c}\delta_{2000} \\
\left(\begin{array}{lll}o & \prime & \prime \prime\end{array}\right)\end{array}$ & $z$ & Identification and Comments \\
\hline$\overline{\mathrm{A}}$ & 002709.8 & -192612.6 & $0.2274 \pm 0.0006$ & "AGN ([OII], [OIII], [Ne III], broad Balmer) \\
\hline $\mathrm{B}$ & 002707.3 & -192636.4 & & M star \\
\hline $\mathrm{C}$ & 002700.5 & -192856.5 & $0.2420 \pm 0.0005$ & galaxy ( $G$-band, $\mathrm{H} \beta, \mathrm{MgIb}, \mathrm{NaId})$ \\
\hline $\mathrm{D}$ & 002659.5 & -192818.6 & $0.2863 \pm 0.0015$ & galaxy $(\mathrm{H}+\mathrm{K}, G$-band, $\mathrm{H} \beta, \mathrm{MgIb})$ \\
\hline g1 & 002712.3 & -193045.5 & $0.2406 \pm 0.0008$ & galaxy $(\mathrm{H}+\mathrm{K}, G$-band, $\mathrm{H} \beta, \mathrm{MgIb}, \mathrm{NaId})$ \\
\hline g2 & 002712.6 & -193043.7 & $0.2380 \pm 0.0012$ & galaxy (CaII-break, $G$-band, $\mathrm{H} \beta, \mathrm{MgIb}, \mathrm{NaId}$ ) \\
\hline g3 & 002712.5 & -193040.1 & $0.2395 \pm 0.0017$ & galaxy (CaII-break, $G$-band, $\mathrm{H} \beta, \mathrm{MgIb}, \mathrm{NaId}$ ) \\
\hline g4 & 002713.1 & -193029.4 & $0.2445 \pm 0.0004$ & galaxy $(\mathrm{H}+\mathrm{K}, G$-band, $\mathrm{H} \beta, \mathrm{MgIb}, \mathrm{NaId})$ \\
\hline g5 & 002713.0 & -193025.6 & $0.2406 \pm 0.0005$ & galaxy $(\mathrm{H}+\mathrm{K}, G$-band, $\mathrm{H} \beta, \mathrm{MgIb}, \mathrm{NaId})$ \\
\hline
\end{tabular}

size of $3.6 \AA /$ pix, and a long-slit of $2.4^{\prime \prime}$ which gives a low spectral resolution of about $24 \AA$. For the reduction of the data we have used the IRAF package (Tody 1993). In the region of the northern X-ray emission we identified 2 objects labeled as A and B in Fig. 3a.

In the region to the west, where the X-ray source 1SAXJ0027.0-1928 is present, we found two galaxies labeled $\mathrm{C}$ and $\mathrm{D}$ in the above mentioned figure. In the region of the southern X-ray emission we obtained spectra for five galaxies which turned out to be members of the cluster. These galaxies are labeled g1 through g5 in Fig. 3b. Table 2 gives the results of the observations:

Based on our imaging and spectroscopic results, we conclude that a blend of the AGN (A) and M-type star (B) X-ray emissions contribute to the extended source 1SAXJ0027.1-1926 to the north. The Abell cluster A33 is the source of the southern X-ray emission 1SAXJ0027.2-1930, while the identification of the source of the western X-ray emission, 1SAXJ0027.0-1928, remains unknown. The two galaxies for which we measured the spectra, and which are the two brightest optical sources in the region, might be responsible for part of the emission of 1SAXJ0027.0-1928, but we need spectroscopic data for more objects to help in the identification. One of the sources (C) is consistent with being part of A33. From the six cluster members listed in Table 2 we obtain for A33 an average $\langle z\rangle=0.2409 \pm 0.0009$, and a very tentative velocity dispersion, given the few cluster galaxies, $\sigma_{\text {los }}=472_{-148}^{+295} \mathrm{~km} \mathrm{~s}^{-1}$. This estimate includes the $1+z$ correction.

\section{X-ray spectroscopy}

The Beppo-SAX concentrator/spectrometer system consists of four separated concentrator mirrors, three of them covering the $1.6-10 \mathrm{keV}$ range (Medium Energy Concentrator Spectrometer, or MECS) and the fourth extending to lower energies down to $0.1 \mathrm{keV}$ (Low Energy Concentrator Spectrometer, or LECS). The concentrators are designed to have a large effective area around the iron $\mathrm{K} \alpha$ line complex: 150 and $50 \mathrm{~cm}^{2}$ for MECS and LECS, at $6 \mathrm{keV}$. Also, Beppo-SAX is able to provide spatially resolved spectra: its energy and angular resolution are $\Delta E / E=8 \%$ at $6 \mathrm{keV}$ and $\theta_{\mathrm{FWHM}} \approx 40^{\prime \prime}$, respectively.

In order to obtain the emission weighted spectral information of the three main sources in the A33 field, we have extracted the photons from circular regions drawn around each source (see Fig. 1). The extraction radius, smaller than the suggested 4 arcmin radius region since the sources are separated by a small angular distance, might introduce a systematic uncertainty. We have used the appropriate Ancillary Response File to correct for this effect. We fitted the source spectra using both a Raymond-Smith code (1977; hereafter RS) or a MEKAL code (Mewe et al. 1995) to model the thermal intracluster gas emissivity and a simple absorbed power-law, nonthermal model. Background spectra have been extracted from library blank-sky images in the same circular regions as the sources.

\section{a) 1SAXJ0027.1-1926}

The spectrum of the brightest source in the field was extracted, both for the LECS and the MECS instruments, from a circular region of 2 arcmin radius centered on the X-ray position of Table 1. The combined LECS-MECS spectrum is shown in Fig. 4: we do not observe any low energy excess absorption in the spectrum, thus we keep $N_{\mathrm{H}}$ fixed at the galactic value of $1.8610^{20} \mathrm{~cm}^{-2}$ (Dickey \& Lockmann 1990) relative to the source position.

The best fit spectral parameters for the MECS spectrum are listed in Table 3 together with their uncertainties at $68.3 \%$ (and $90 \%$ in parentheses) confidence level. We use 605 source photons in this spectral fit.

Within 2 arcmin from its center, the source has a flux of $F_{2-10 \mathrm{keV}}=(4.20 \pm 0.32) 10^{-13} \mathrm{erg} \mathrm{s}^{-1} \mathrm{~cm}^{-2}$, evaluated using the MEKAL best fit parameters. The other models give similar fluxes. This flux is also consistent, within the errors, with the flux of the X-ray source 1RXSJ002709.5 - 192616 in the ROSAT band.

The optical magnitude of the M-star if $m_{V} \approx 19$. Assuming that the X-ray flux of the M-star contributes to $50 \%$ of the total flux of 1SAXJ0027.1-1926, we obtain $F_{2-10} / F_{V} \approx 2$. in the $2-10 \mathrm{keV}$ band and $F_{0.3-3.5} / F_{V} \approx$ 
Table 3. 1SAXJ0027.1-1926

\begin{tabular}{ccccccc}
\hline \hline Model & pho. index & $z$ & $T$ & bins & $\chi^{2}$ & $\chi_{\text {red }}^{2}$ \\
\hline & & & $\mathrm{keV}$ & & & \\
\hline \hline RS & - & $0.245 \pm 0.023$ & $3.99_{-0.75(+1.83)}^{+0.97(-0.98)}$ & 61 & 56.12 & 0.97 \\
MEKAL & - & $0.245 \pm 0.024$ & $3.90_{-0.69(-0.98)}^{+0.96(-1.97)}$ & 61 & 55.98 & 0.97 \\
PL & $2.05_{-0.14(-0.18)}^{+0.17(+0.41)}$ & - & - & 61 & 62.55 & 1.06 \\
\hline \hline
\end{tabular}

Table 4. 1SAXJ0027.2-1930

\begin{tabular}{ccccccc}
\hline \hline Model & pho. index & $z$ & $T$ & bins & $\chi^{2}$ & $\chi_{\text {red }}^{2}$ \\
\hline & & \multicolumn{5}{c}{$\mathrm{keV}$} \\
\hline RS & - & 0.2409 & $2.91_{-0.54(-0.83)}^{+1.25(+2.44)}$ & 19 & 20.43 & 1.28 \\
MEKAL & - & 0.2409 & $2.88_{-0.55(-0.83)}^{+1.23(+2.46)}$ & 19 & 20.45 & 1.28 \\
PL & $2.67_{-0.37(-0.57)}^{+0.41(+0.67)}$ & - & - & 19 & 22.05 & 1.30 \\
\hline \hline
\end{tabular}

Table 5. 1SAXJ0027.0-1928

\begin{tabular}{ccccccc}
\hline \hline Model & pho. index & $z$ & $T$ & bins & $\chi^{2}$ & $\chi_{\text {red }}^{2}$ \\
\hline & & \multicolumn{5}{c}{$\mathrm{keV}$} \\
\hline \hline RS & - & $0.36 \pm 0.09$ & $2.56 \pm 0.86$ & 13 & 14.70 & 1.47 \\
MEKAL & - & $0.35 \pm 0.11$ & $2.45 \pm 0.85$ & 13 & 14.79 & 1.48 \\
PL & $2.63 \pm 0.60$ & - & - & 13 & 14.98 & 1.36 \\
\hline \hline
\end{tabular}

6 in the $0.3-3.5$ energy band (assuming a thermal emission at $T=1 \mathrm{keV}$ ). This ratio is almost one order of magnitude higher than the values of $F_{0.3-3.5} / F_{V}$ for X-ray selected stars in the EMSS (see Fig. 1 in Maccacaro et al. 1988). This means that the contribution of the Mstar to the X-ray flux of 1SAXJ0027.1-1926 should be $\lesssim 8 \%$ to be consistent with the values of $F_{\mathrm{X}} / F_{V}$ for normal stars. If this is the case, then more than half of the X-ray emission of 1SAXJ0027.1-1926 is due to the AGN (listed as $\mathrm{A}$ in Table 2) at $z=0.2274$ with a luminosity $L_{2-10 \mathrm{keV}} \lesssim 4.510^{43} \mathrm{erg} \mathrm{s}^{-1}$. Otherwise, the source 1SAXJ0027.1-1926 should result from the blend of the AGN and of a different unknown X-ray source.

\section{b) $1 S A X J 002 \% .2-1930$}

The average spectrum of 1SAXJ0027.2-1930 was extracted from a circular region of 2 arcmin radius centered on the X-ray position of Table 1 (see also Fig. 5). In this region there is a clear excess of galaxies (see Fig. 3) which is $\sim 1.5$ arcmin away from the Abell catalog position of A33. Fitting the spectrum (which contains 140 source photons) with a RS thermal model with temperature, abundance and redshift as free parameters, the fit gives $\chi_{\text {red }}^{2}=$ 1.22. Fixing the value of $N_{\mathrm{H}}$ to the galactic value (1.86 $10^{20} \mathrm{~cm}^{-2}$ ) we obtain an average temperature $T=3.1 \pm$ $0.9 \mathrm{keV}$ and a redshift $z_{\mathrm{X}}=0.72 \pm 0.04$. The abundance is only marginally constrained at $\mathrm{Fe} / \mathrm{H}=0.98 \pm 0.71$ of the solar value. However, the fit results are mainly due to a marginally significant spectral feature at $E \sim 4 \mathrm{keV}$.
Therefore, we fixed the redshift of the X-ray source at $z=0.2409$, as measured from the optical spectra (see Sect. 3), and we fitted the spectrum again, fixing the abundance to a value $\mathrm{Fe} / \mathrm{H}=0.3$ solar. The results of the fit are shown in Table 4 . Uncertainties in the temperature of 1SAXJ0027.2-1930 are given at $68.3 \%$ (and 90\% in parentheses) confidence level. The low count rate of the source does not allow a more accurate description of the $\mathrm{X}$-ray emission.

Assuming the MEKAL best fit parameters we obtain an integrated flux of $F_{2-10 \mathrm{keV}}=(2.4 \pm 0.3) 10^{-13} \mathrm{erg}$ $\mathrm{s}^{-1} \mathrm{~cm}^{-2}$ in the 2 arcmin radius extraction region (which corresponds to a linear size of $\approx 1 h_{50}^{-1} \mathrm{Mpc}$ ). The other models give consistent fluxes. At the redshift of the cluster this flux corresponds to a luminosity $L_{2-10 \mathrm{keV}}=$ $(7.7 \pm 0.9) 10^{43} h_{50}^{-2} \mathrm{erg} \mathrm{s}^{-1}$ and to a bolometric luminosity $L_{\mathrm{bol}}=(2.2 \pm 0.3) 10^{44} h_{50}^{-2} \mathrm{erg} \mathrm{s}^{-1}$.

\section{c) $1 S A X J 002 \% .0-1928$}

We extracted the spectrum of 1SAXJ0027.0-1928 from a circular region of 1 arcmin radius centered on the X-ray position of Table 1 (see Fig. 6). Results of the fit are shown in Table 5 (uncertainties on the best fit values are given here at $68.3 \%$ confidence level. Note that the spectrum of this source contains 90 source photons). Assuming an absorbed power-law non-thermal model, we derived a flux of $F_{2-10 \mathrm{keV}}=(4.74 \pm 0.8) 10^{-14} \mathrm{erg} \mathrm{s}^{-1} \mathrm{~cm}^{-2}$. There are two galaxies, a spiral (C) at the same $z$ of A33, and 


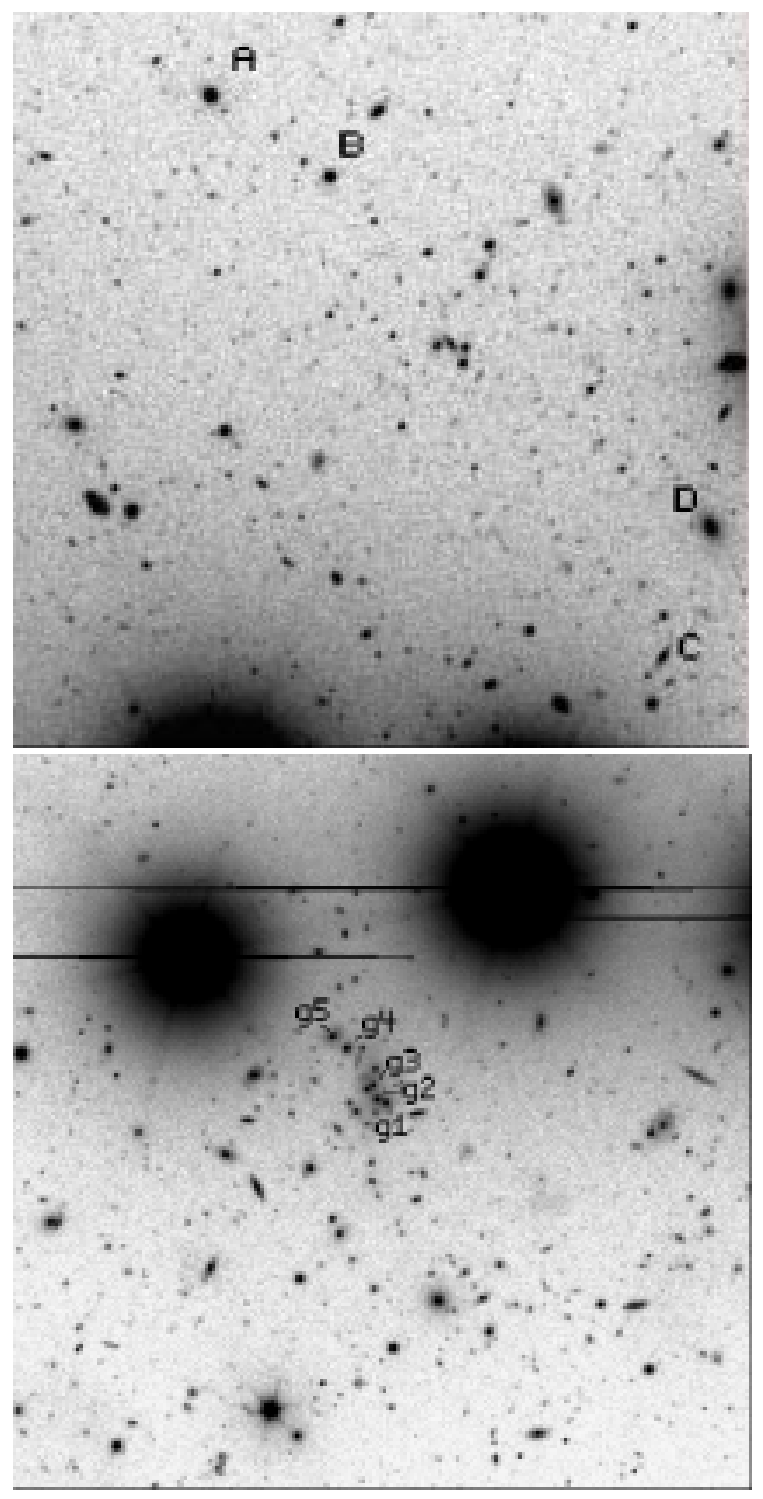

Fig. 3. The two images are $1024 \times 1024(3.7 \times 3.7$ arcmin $)$ subarrays extracted from two $B$-band exposures taken at the Keck II telescope. The image to the top shows the field around 1SAXJ0027.1-1926 and 1SAXJ0027.0-1928 and the image to the botton shows the field around 1SAXJ0027.2-1930. North is up and East to the left

an elliptical (D) in the region for which we took an optical spectrum.

The identification of the source is not certain at the moment. Assuming that the galaxy D at $z=0.2863$ is the $\mathrm{X}$-ray emitter, its X-ray luminosity would be $L_{2-10 \mathrm{keV}}=$ $1.910^{43} \mathrm{erg} \mathrm{s}^{-1}$. Such an X-ray luminosity seems to be sensibly higher than the X-ray luminosity of a "normal" galaxy. The possibility that the $\mathrm{X}$-ray emission is due to a more distant, unidentified object cannot be excluded at present.

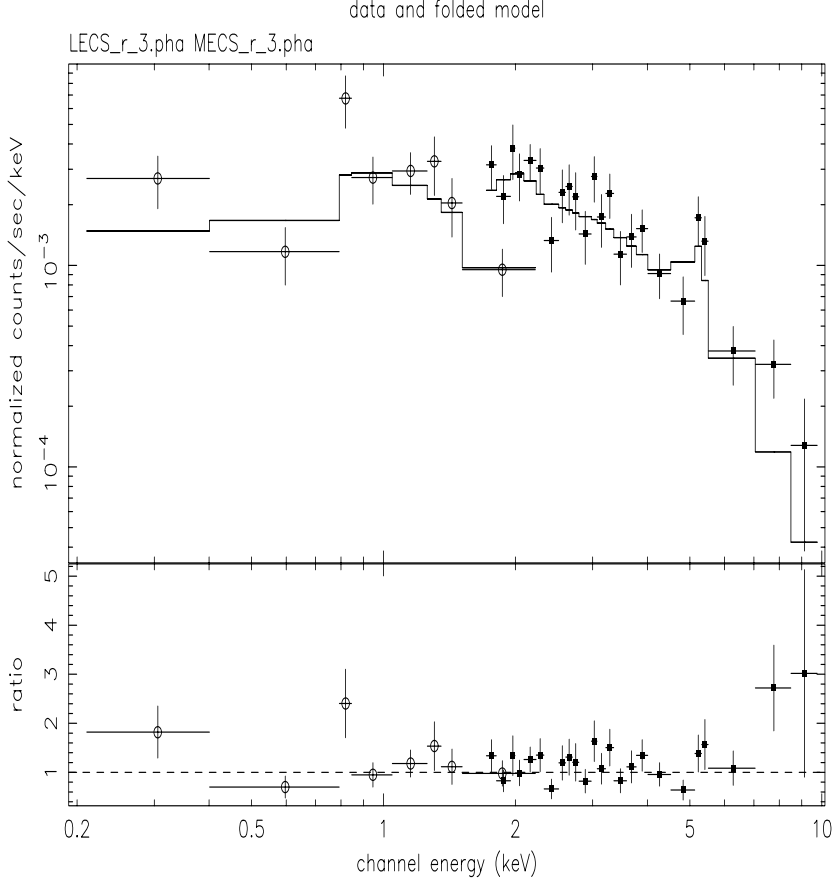

Fig. 4. The combined LECS and MECS spectrum of the source 1SAXJ0027.1-1926 extracted from a 2 arcmin radius regions. The spectrum shown in figure has been rebinned so that the significance of each bin is at least $3 \sigma$. The best fit model is a MEKAL thermal model (see text for details). The spectrum has been further rebinned using XSPEC for graphical purposes

\section{Discussion}

In this paper we presented the first detailed X-ray observation of the distant Abell cluster A33, obtained with the Beppo-SAX satellite. We have closely examined and clarified the complex X-ray emission in the direction of A33. The analysis of the X-ray data revealed the presence of three different X-ray sources in the field of A33. The X-ray counterpart of the cluster is 1SAXJ0027.2-1930. We present a spectroscopic redshift for A33, applying a $\sim 20 \%$ correction to the previous photometric estimate. From optical spectra of six cluster galaxies we measure a redshift $z=0.2409 \pm 0.0009$ and a velocity dispersion along the line of sight $\sigma_{\mathrm{los}}=472_{-148}^{+295} \mathrm{~km} \mathrm{~s}^{-1}$. The dominant X-ray component (incorrectly linked with A33 in the past) is associated with a blend of an AGN and M star, while the X-ray emission from A33 is $\sim 4$ times fainter. Using the proper X-ray flux and measured redshift, we determine a more realistic cluster luminosity of $L_{2-10 \mathrm{keV}}=(7.7 \pm 0.93) 10^{43} h_{50}^{-2} \mathrm{erg} \mathrm{s}^{-1}$, one to two orders of magnitude lower than previous attempts. The MECS spectral resolution also allows us to determine that the intracluster gas temperature is $T=2.91_{-0.54}^{+1.25} \mathrm{keV}$. No useful information on the cluster abundance is given due to the low count rate of the source in the MECS detector. 

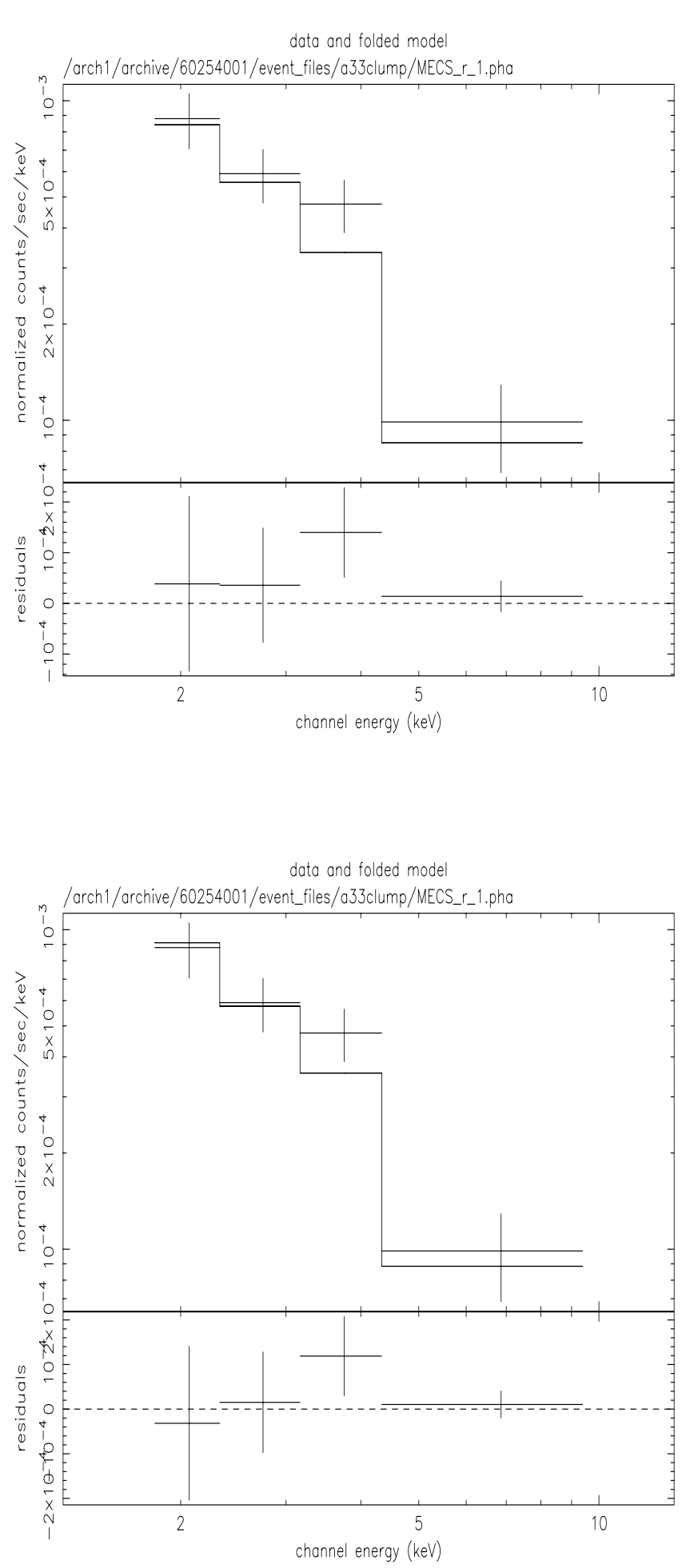

Fig. 5. The SAX MECS spectra of 1SAXJ0027.2-1930 fitted with a thermal MEKAL model (upper panel) and with an absorbed power-law model (lower panel). Details of the spectral analysis are given in Table 4 . The spectrum has been further rebinned using XSPEC for graphical purposes

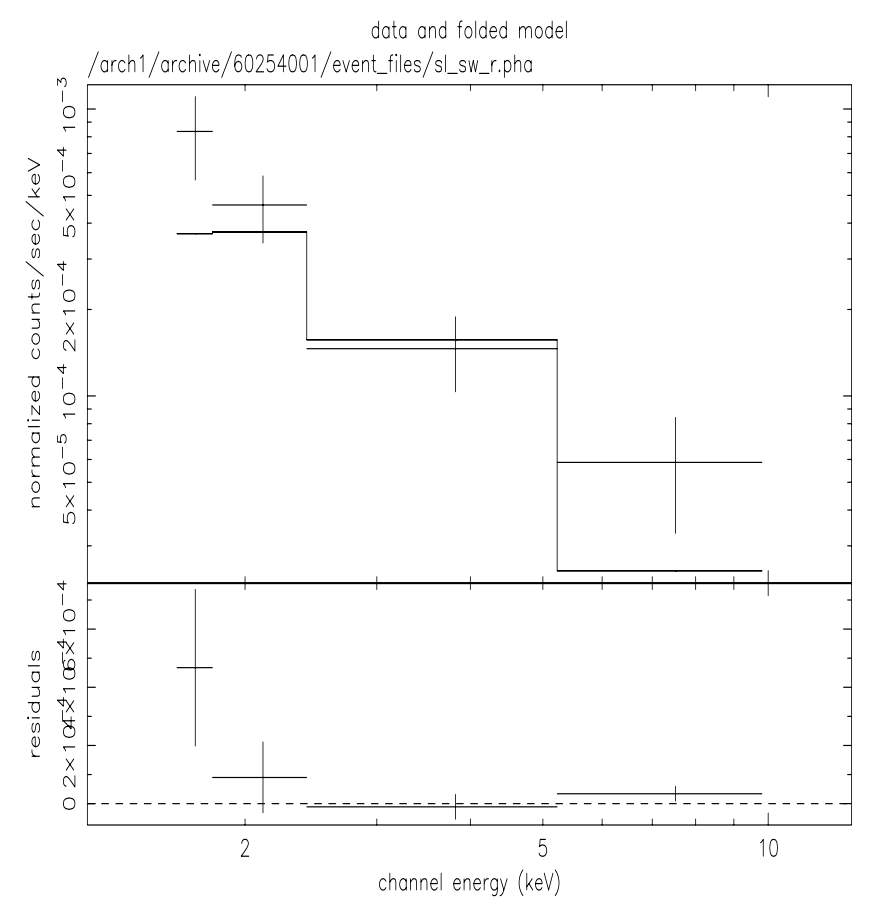

Fig. 6. The SAX MECS spectrum of 1SAXJ0027.0-1928 fitted with a non-thermal power-law model (see Table 4 for details). The spectrum has been further rebinned using XSPEC for graphical purposes

In the following we will focus on measured quantities such as the low temperature and low velocity dispersion. We are dealing here with a moderately rich $(R=1)$ and distant $(D=3)$ Abell cluster but with X-ray luminosity and temperature more typical of nearby $(z<0.1)$ poor clusters. The temperature of A33 is commensurate with the predictions from its X-ray luminosity from the $L_{\mathrm{X}}-T$ relation by David et al. (1993) and Arnaud \& Evrard (1999). There is an extensive literature on the correlation between these two basic and measurable quantities (Edge \& Stewart 1991; Ebeling 1993; David et al. 1993; Fabian et al. 1994; Mushotzky \& Scharf 1997; Markevitch 1998; Arnaud \& Evrard 1999). Comparing the bolometric luminosity of A33 with the best fit relation, $\log \left(L_{\mathrm{X}}\right)=(2.88 \pm$ $0.15) \log (T / 6 \mathrm{keV})+(45.06 \pm 0.03)$ obtained by Arnaud \& Evrard (1999), analyzing a sample of 24 low- $z$ clusters with accurate temperature measurements and absence of strong cooling flows, we would expect for the A33 a temperature of $3.4 \mathrm{keV}$, as compared with our deduced value $2.9_{-0.54}^{+1.25}$. The $L_{\mathrm{X}}-T$ relation does not seem to evolve much with redshift since $z=0.4$ (Mushotzky \& Scharf 1997). Note however that the ASCA data that they use show a strong bias at the low-luminosity end of the distribution due to the absence of objects in the lower luminosity range in the ASCA database. The present data on a cluster at about 0.2 are thus important to fill in the gap in the 
$L_{\mathrm{X}}-T$ relationship found among rich clusters and groups (see Mushotzky \& Scharf 1997).

The measured velocity dispersion of A33 is also commensurate with the predictions from the $\sigma-T_{\mathrm{X}}$ relationship. A large number of authors (see Table 5 in Girardi et al. 1996, or Table 2 in Wu et al. 1998, for an exhaustive list of papers on the subject) have attempted to determine the $\sigma-T$ using different cluster samples in order to test the dynamical properties of clusters. Girardi et al. (1996) have derived a best fit relation between the velocity dispersion and the X-ray temperature, with more than $30 \%$ reduced scatter with respect to previous work (Edge \& Stewart 1991; Lubin \& Bahcall 1993; Bird et al. 1995; Wu et al. 1998, among others). If we substitute the temperature of 1SAXJ0027.2-1930 in the best fit relation $\log (\sigma)=(2.53 \pm 0.04)+(0.61 \pm 0.05) \log (T)$, derived by Girardi et al. (1996) a value of $650 \mathrm{~km} \mathrm{~s}^{-1}$ would be expected for the 1-D velocity dispersion, somewhat higher but within the uncertainties of the measured value from six cluster members of A33. If we assume energy equipartition between the galaxies and the gas in the cluster $(\beta=$ 1 ) and we use the measured temperature of $2.9 \mathrm{keV}$ from the SAX data in the equation $\beta=\mu \mathrm{m}_{\mathrm{p}} \sigma_{v}^{2} / k T_{\text {gas }}$ (where $\mu \mathrm{m}_{\mathrm{p}}=0.62$, for solar abundance), we obtain a velocity dispersion of $665 \mathrm{~km} \mathrm{~s}^{-1}$.

The data for A33 are also consistent with the relation $\sigma_{\text {los }} \propto(T / \mathrm{keV})^{0.6 \pm 0.1}$ found by Lubin \& Bahcall (1993) and increase its statistical significance in the low temperature $(T \lesssim 3 \mathrm{keV})$ range and at intermediate redshifts $(z \sim 0.2)$ where only a few clusters have measured values of $\beta$. This issue will be discussed in a forthcoming paper.

We have also found that the bright source 1SAXJ0027.1-1926 has an extended appearance which is due to the blending of two different sources: an AGN at $z=0.227$ and approximate $B$ magnitude $M_{B} \approx-23.9$ (derived from the apparent $B$ magnitude as given in the APM scans) and an M-type star. The X-ray spectrum does not show any line features, and it is contaminated by the emission of the M star. Given the low statistics we did not try to disentangle the two contributions but we consider an upper limit to the AGN emission using the $F_{\mathrm{X}} / F_{V}$ for the $\mathrm{M}$ star. The ROSAT BSC source found at a position consistent with the coordinates of 1SAXJ0027.1-1926 is most probably associated with the AGN. The distance between the foreground AGN and the cluster is $\Delta d_{\mathrm{L}} \approx 89.2 h_{50}^{-1} \mathrm{Mpc}$. At the redshift of the AGN, the observed total flux corresponds to a luminosity $L_{\mathrm{X}} \lesssim 4.510^{43} \mathrm{erg} / \mathrm{s}$, which can be considered as an upper limit to the AGN luminosity.
We also detected a point-like faint source, 1SAXJ0027.0-1928, for which no X-ray spectroscopic identification was possible. The $2-10 \mathrm{keV}$ spectrum of this source can be fitted by both thermal and non-thermal models (see Table 5) but we do not elaborate further given the poor statistics.

Acknowledgements. S.C. acknowledges useful discussions with G. Hasinger and C. Sarazin. Partial financial support from ASI, NASA (NAG5-1880 and NAG5-2523) and NSF (AST95-00515) grants is gratefully acknowledged. We appreciate the generosity of B. Tully who allowed us to take some images and spectra during his observing runs.

\section{References}

Abell G.O., 1958, ApJS 3, 211

Abell G.O., Corwin H.G., Olowin R.P., 1989, ApJS 70, 1

Arnaud M., Evrard A.E., 1999, MNRAS 305, 631

Arnaud M., Lachieze-Rey M., Rothenflug R., Yamashita K., 1991, A\&A 243, 56

Bird C.M., Mushotzky R.F., Metzler C.A., 1995, ApJ 453, 40

Boella G., et al., 1997a, A\&AS 122, 299

Boella G., et al., 1997b, A\&AS 122, 327

Dickey J.M., Lockman F.J., 180, ARA\&A 28, 215

David L.P., Slyz A., Jones C., Forman W., Vrtilek S.D., 1993, ApJ 412, 479

Ebeling H., 1993, Ph.D. Thesis, MPE

Edge A.C., Stewart G.C., 1991, MNRAS 252, 414

Fabian A.C., Crawford C.S., Edge A.C., Mushotzky R.F., 1994, MNRAS 267, 779

Giommi P., Angelini L., Jacobs P., Tagliaferri G., 1991, in "Astronomical Data Analysis Software and Systems I", Worrall D.M., Biemesderfer C. and Barnes J. (eds.) ASP Conf. Ser. 25, 100

Girardi M., Fadda D., Giuricin G., Mardirossian F., Mezzetti M., 1996, ApJ 457, 61

Johnson M.W., et al., 1983, ApJ 266, 425

Leir A.A., Van Den Bergh S., 1977, ApJS 34, 381

Lubin L.M., Bahcall N.A., 1993, ApJ 415, L20

Kowalski M.P., et al., 1984, ApJS 56, 403

Maccacaro T., Gioia I.M., Wolter A., Zamorani G., Stocke J.T., 1988, ApJ 326, 680

Markevitch M., 1998, ApJ 504, 27

Mewe R., Kaastra J., Liedahl L., 1995, Legacy 6, 16

Mushotzky R.F., Scharf C.A., 1997, ApJ 482, L13

Oke J.B., Cohen J.G., Carr M., et al., 1995, PASP 107, 375

Raymond J.C., Smith B.W., 1977, ApJS 35, 419

Tody D., 1993, "IRAF in the Nineties" in Astronomical Data Analysis Software and Systems II, Hanisch R.J., Brissenden R.J.V., \& Barnes J. (eds.), ASP Conf. Ser. 52, 173

Voges W., et al., 1996 IAUC 6420

Wu X.-P., Fang L.-Z., Xu W., 1998, A\&A 338, 813 